\title{
Exoskeleton-Based Exercisers for the Disabilities of the Upper Arm and Hand
}

\author{
${ }^{1}$ Ioannis Sarakoglou, ${ }^{1}$ Sophia Kousidou, ${ }^{2}$ Nikolaos G. Tsagarakis and \\ ${ }^{2}$ Darwin G. Caldwell \\ University of Salford', Manchester, UK \\ Italian Institute of Technology2, Genoa, Italy
}

\section{Introduction}

The impact of disability on society is great not only on direct treatment costs. Invaluable loss of human creative activity and mental wellbeing as well as productivity losses reflect the indirect impact on the disabled individual as well as on society as a whole.

Stroke is the leading cause of disability in the industrialised countries. Every year, over 130,000 people in the U.K. suffer strokes, with 13,000 under retirement age. Ischemia or haemorrhage in the brain may be the cause of cerebral vascular accidents which result in strokes (Parker et al., 1986). Fortunately over $65 \%$ of patients survive but the majority does have residual disabilities with up to $1 / 3$ having severe disabilities particularly in the upper limb and hand. Hemiplegia, the most common impairment resulting from stroke, leaves the survivor with a stronger unimpaired arm and a weaker impaired one (hemiparesis). Traumatic injuries as well as conditions like muscular dystrophy, arthritis and regional pain syndromes, also add to the major causes of disability and functional dependence. Deficits in $\varepsilon$ motor control and coordination synergy patterns, spasticity and pain are some of the most סे common symptoms of these conditions (Parker et al., 1986).

$\underset{0}{0}$ In the case of stroke victims, it is widely accepted that spontaneous recovery accounts for the motor and functional restoration taking place within the first months after the stroke incident.

으 Recent evidence has shown that further improvement can be achieved if neural organisation is O modified. Partially damaged neural pathways can be reinstituted and neurons not normally $\stackrel{\Phi}{. \pm}$ involved in an activity can be engaged. Neuroplasticity is use-dependent; therefore it has been shown that intensive and repetitive physiotherapy may be necessary to modify neural organization (Carr \& Shepherd, 1987) and recover functional motor skills. In the case of other ( disability victims, repetitive physiotherapy is also the key for regaining motor control, as it đI contributes in regaining muscle strength as well as in restoring the joints' range of motion.

을 Despite the benefits of intensive physiotherapy, upper limb and hand disability are seldom సँ considered life-threatening; therefore they rate relatively low on the priority list for urgent on medical assistance. In addition to that, manipulative physiotherapy procedures are labour\& intensive with hundreds of arm flexing movements per day forming part of a rehabilitation o regime that is no untypical. Manipulation requires high levels of one to one attention from

highly skilled medical personnel, but there is an international shortage of physiotherapists.

Ф Finally, patients must receive individualised treatment. The need for longer treatment O periods, more intensive regimes and the shortage of trained stuff means that robotic and 
power assistive techniques are increasingly viewed as a potential replacement for the physical labour leaving the therapists with greater time to develop the treatment plan. Computer generated three-dimensional environments (VEs) can provide visual, auditory and physical (haptic) interactions in a way that engages a patient's attention while at the same time keeping him/her motivated. Motivation is a key factor in successful rehabilitation. If an impaired person lacks motivation, he/she may use the unimpaired arm/hand in performing activities of the daily living (ADLs) and therefore hamper the functional restoration of the impaired arm/hand (Nakayama et al., 1994). The role of VEs in rehabilitation can be considered as dual: they provide the therapists with a set-up for repetitive functional ADL training while at the same time giving quality feedback to the patients helping them control their physiological responses in an engaging and entertaining way.

There has been a lot of work on power-assisted device therapy and as a result, there is an increasingly wide and diverse range of systems. These systems range from simple powered 2-link orthoses to industrial robots and from simple data gloves in VEs to complicated hand exoskeletons. They use a variety of actuation methods and control strategies and they are targeted at different disabilities.

The next two sections explore the art in rehabilitation exoskeletons for the upper arm and hand. Sections 4 and 5 present work that is undergoing at the University of Salford. More specifically, Section 4 presents a rehabilitation system using Salford Rehabilitation Exoskeleton as a medium for delivering therapy whereas Section 5 presents a Hand Rehabilitation Exoskeleton. Section 6 concludes with a brief discussion including the authors' view regarding future directions in the area of Rehabilitation Robotics.

\section{Upper Arm Rehabilitation Exoskeletons}

The major findings in robot-mediated rehabilitation come from two systems that have undergone extensive clinical trials: the MIT-MANUS robot (Hogan et al., 1992; Volpe et al., 2000) and the Palo Alto/VA Stanford Mirror Image Motion Enabler (MIME) (Burgar et al., 2000). Due to the fact that both systems are using robots rather than exoskeletons to deliver therapy to stroke patients, they will not be the subject of detailed presentation here. The main findings of these clinical trials however, indicated a significant improvement in patients' motor abilities while there was no significant improvement in their functional skills.

The orthoses/exoskeleton systems presented below are targeted mostly at patients with muscular weakness or multiple sclerosis. Some of them have been clinically tested but none of them has undergone extensive clinical trials.

\subsection{ARMin}

ARMin (Mihelj et al., 2006) is a 6 DOF exoskeleton developed at the Swiss Federal Institute of Technology in Zurich. It is specifically designed for neurological rehabilitation; as a device-therapy medium as well as a tool to test existing rehabilitation strategies and find the best rehabilitation practice. ARMin is a semi-exoskeleton solution in the sense that its structure is fixed on the wall via an aluminium frame and the patient's wheelchair can be placed beneath figure. 2.1. Its kinematic structure is depicted in figure 2.2. The exoskeleton has $3 \mathrm{DOF}$ at the shoulder permitting horizontal, vertical and internal/external shoulder 
rotation, 1 DOF for elbow flexion extension, 1 DOF for forearm pronation/supination and finally, $1 \mathrm{DOF}$ for wrist flexion/extension.

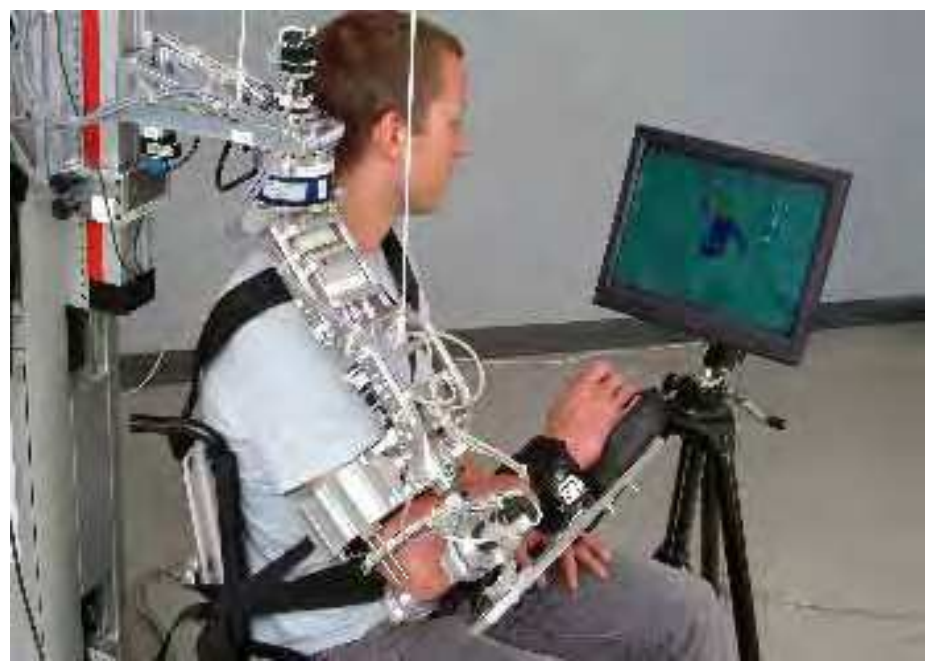

Fig. 2.1. ARMin. The image depicts the semi-exoskeleton structure. (Mihelj et al., 2006).

Impedance control is used to ensure compliant behaviour and many safety features have been incorporated in order not pose danger to the patient in case of malfunction. Its modes of operation are currently three. In the movement therapy mode, the therapist guides the patient's arm to form trajectories which can be repeated by the exoskeleton with different velocities. This mode is targeted at preserving joint range of motion and preventing joint degeneration. The game therapy mode strives to motivate the patient with simple games such as catching a virtual ball. If the patient is able to play the game, ARMin just compensates its weight. If the patient cannot play the game then it guides the patient's arm with an adjustable force towards the ball position. Finally, in the ADL training mode, the patient can train in ADL tasks like eating or grasping. In this mode the patient generates the trajectory in the sense that based on the patient's position and speed, ARMin predicts the required forces and torques.

In a pilot study with ten healthy subjects and five patients, comfort, functionality and acceptance was tested out. During the movement therapy, trajectory recording and repetition at different velocities was well performed and the robot support for the game therapy mode was adequate. The subjects assigned a grading of 8.5 to the therapy modes and an increase in their performance was noted progressively.

\subsection{Wearable Orthosis for Tremor Assessment and Suppression (WOTAS)}

WOTAS (Ruiz et al., 2006) is an upper limb exoskeleton specifically designed to measure and compensate for movement disorders such as tremor. It is actuated by electric motors at the wrist and elbow and its sensory system comprises of chip gyroscopes (which measure tremor force constantly) and kinetic sensors. The total weight of the system is roughly 850 gr. Impedance control strategy is used and real-time filtering algorithms distinguish between intended motion and tremor. 


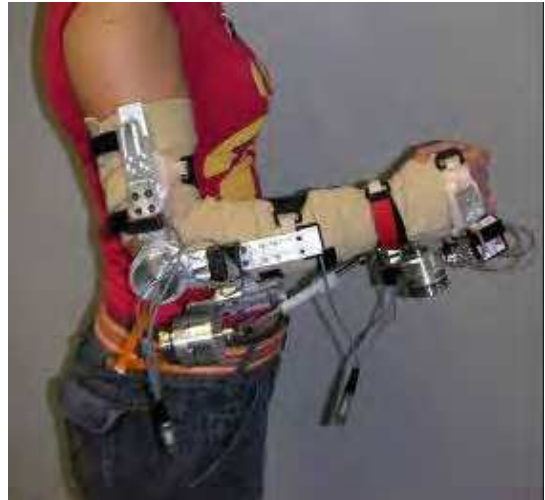

(a)

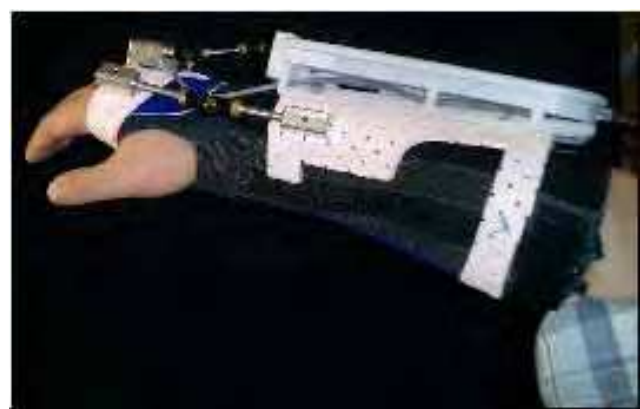

(b)

Fig. 2.2. WOTAS. Image (a) shows a subject wearing the exoskeleton (Ruiz et al., 2006) while image (b) shows the forearm module.

Tremor is suppressed with the means of an actuator based on magneto-rheological fluids (whose viscosity can change by applying a magnetic field and therefore act as an effective damper).

Initial studies were performed with subjects wearing the exoskeleton while executing various tasks of the daily living. It was reported that WOTAS did not affect the subjects' range of motion. At the second stage of the study, the system added viscosity and inertia in order to suppress tremor (passive control strategy) and was able to estimate and measure tremor parameters. It was estimated that the system could suppress $30 \%$ of the production of tremor power. The reduction of the tremor power was sustained in the order of $80 \%$ in patients with severe tremor.

\subsection{Motorized Upper Limb Orthotic System (MULOS)}

MULOS (Motorized Upper Limb Orthotic System) (Johnson et al. 2001) was developed under a project funded by the Technology Initiative for Disabled and Elderly (TIDE) program of the Commission of European Communities and it was intended as stroke rehabilitation as well as an assistive. MULOS is a 5 DOF powered orthosis for the upper limb which allows the movement of the shoulder (3 DOF), the elbow and the forearm. It was designed to provide single joint exercise and operates in 3 modes:

a) Assistive, to compensate for loss of muscular action caused, for instance, by muscular dystrophy of high-level spinal cord injury.

b) Continuous Passive Motion, to provide physical therapy to selected joints of the arm.

c) Exercise, to provide graded resistance in order to allow exercise therapy to people with muscle weakness.

The shoulder structure is a 3 DOF mechanism having intersecting axes to allow it to behave as a spherical joint with a centre approximate coincident with that of the user's shoulder. The structure has sufficient compliance to allow a full range of motion at the shoulder. The joints are powered by cable drives in such a way as to keep the electric motors as close to the first joint as possible and thus, keep required torques to a minimum. 
The elbow joint has one degree of freedom providing flexion/extension and a separate power unit positioned at the wrist provides elbow pronation/supination. The system can achieve a maximum output torque of $7 \mathrm{Nm}$ and maximum speed of $9.5 \mathrm{rpm}$. An integral slip clutch between the motor and the elbow drive ensures safety of operation. A potentiometer for position control is located in line with the lower arm section of the orthosis.

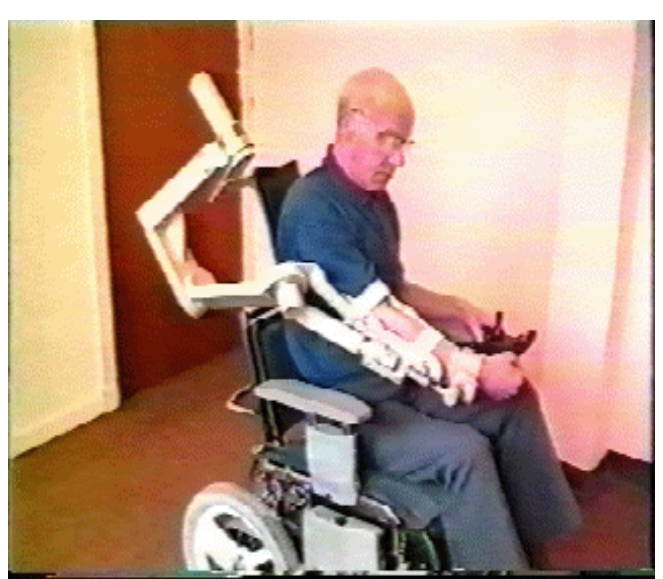

(a)

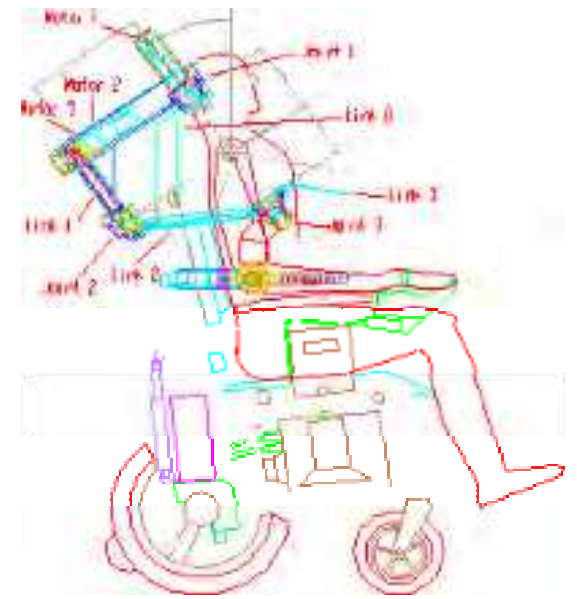

(b)

Fig. 2.3. Motorised Upper Limb Orthotic System (MULOS). (a) shows a patient wearing the exoskeleton (b) a rough sketch of the position of motors, joints and links (Johnson et al., 2001).

Control hardware is mounted at the back of the wheelchair and the available control strategies are:

a) Point-to-point, using a 4 DOF joystick with the unimpaired limb to control the movement of the impaired limb

b) Joint-by-joint control

c) Walk-through programming in which trajectories can be pre-programmed moving the orthosis through a particular trajectory on a passive mode.

Although the device seemed to have good potentials, its development stopped in 1997.

\subsection{FUNCTIONAL UPPER ARM ORTHOSIS}

Functional Upper Limb Orthosis (Rahman et al., 2000) was a joint project between Alfred I. DuPont Hospital for Children and the School of Biomedical Engineering, Science and Health Systems at Drexel University. It was targeted at people with limited strength in their arms as a result of muscular dystrophy, spinal muscular atrophy and partial spinal cord injury.

The goal of the particular orthosis was to provide a sense of "floatation" that would allow a person with neuromuscular weakness to move his/hers arms. This was accomplished by gravity-balancing the entire arm - hand, forearm and upper arm - for all positions in 3D space. It had four DOF - two at the shoulder and two at the elbow. Three prototypes have been developed and evaluated 
The orthosis had been tested on 10 patients. It was mounted on an adjustable stand and was placed so that the subject's shoulder joint was just above the anatomical shoulder. The patients were asked to put their right arm in the orthosis trough and a Velcro strap then secured the arm. The orthosis bungee cords were then stretched or relaxed based on their observed "floatation". After being able to move their arm at their own will, they were asked to perform activities of daily living. The responses from the patients varied during this first series of trials but the functionality of the device as far as the size adjustability is concerned was expressed repeatedly by all patients.

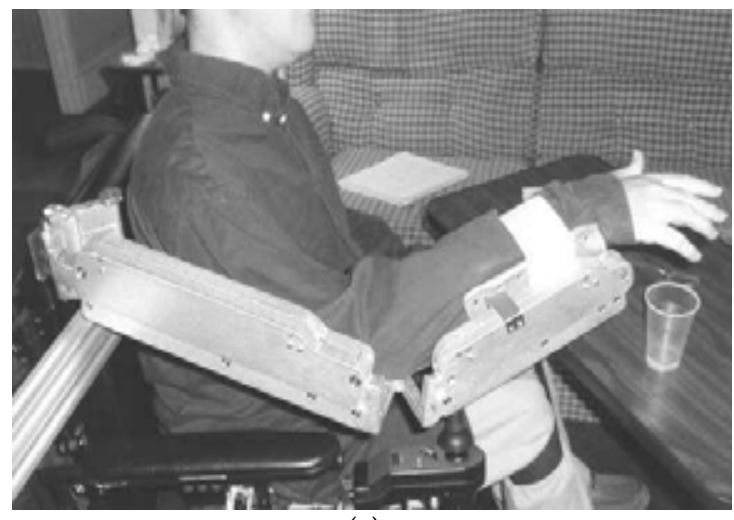

(a)

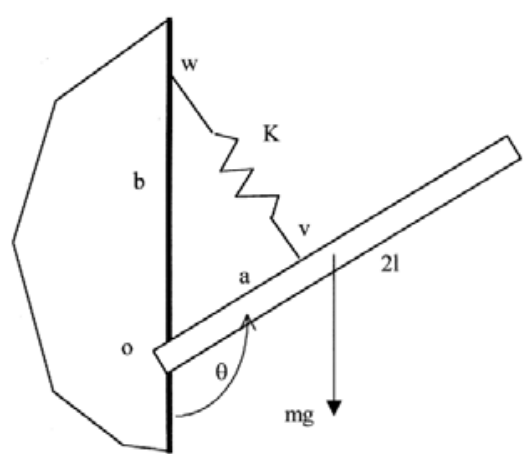

(b)

Fig.2.4. Functional Upper Arm Orthosis. (a) shows a patient wearing the exoskeleton (b) depics the gravity compensation model (Rahman et al., 2000).

Due to the inexact gravity compensation (in vertical movement), the device is rarely prescribed. The majority of the BFO users settle only for planar motion and rely on compensatory body movements to achieve vertical motions.

\subsection{ROBOTIC ASSISTANT OF UPPER LIMB EXERCISE}

Alastair Cozens, while he was with the Rheumatology and Rehabilitation Research Unit at the University of Leeds, performed a study in which a robot assisted an active single limb exercise (Cozens, 1999). Robot assistance was demonstrated using torque applied to an individual joint, with electromyographic (EMG) data to confirm active exercise and monitor the pattern of activity within the antagonistic muscle pair.

During the experiments, the patient sat with the shoulder flexed forward to 90 degrees. The forearm was fastened to a lever, which could rotate in the horizontal plane about an axis aligned with the elbow. The upper arm was immobilized so that the lever could only be moved by elbow flexion/extension. Around the lever circumference target lamps were placed at locations corresponding to 10 degrees and 80 degrees elbow flexion denoting the targets towards which the lever should be aimed during extension and flexion. The angular movement of the lever was monitored by an electrogoniometer and an accelerometer whereas EMG activity in biceps and triceps was monitored via surface electrodes. The lever was set into motion by means of a servomotor, which assisted flexion/extension of the elbow. The motor had a maximum preset torque of $2 \mathrm{Nm}$ for safety reasons. 


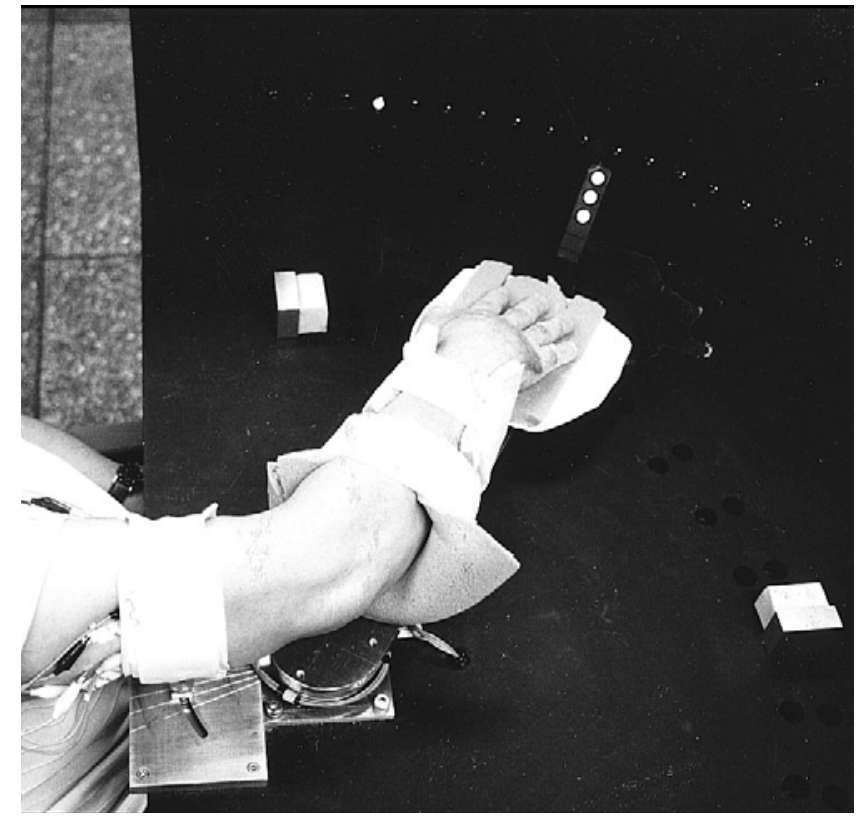

Fig. 2.5. Robotic Assistance of Upper Limb Exercise. The image depicts the orthoses, the contact points with the arm and the target towards which the lever should be aimed during extension and flexion of the elbow (Cozens, 1999).

Experiments using this arrangement were performed on ten stroke and multiple sclerosis patients of ages between 47 and 69. Each of these patients exhibited a weakness of the upper limb such as they could move the lever a little but were unable to complete an unassisted ten-cycle exercise with full movement between target lamps on every cycle. Some patients exhibited spasticity. During a ten cycle experiment, it was observed that the patients completed two cycles with extreme effort and biceps spasticity offered resistance to the elbow movement. When the motor assisted the elbow extension, the patients were able to achieve a full extension/flexion range in all ten cycles.

\subsection{BALANCED FOREARM ORTHOSIS}

Balanced Forearm Orthosis (Alexander et al., 1992) is a body-powered device that was developed in 1965. It was designed for people with muscular weakness and provided with the ability to move their arms in a horizontal plane.

Movement was accomplished via two linkages having joints along the vertical axes. One end of the orthosis was mounted on a wheelchair and the other end was connected to a trough into which a person placed his/her forearm. In the cases where the shoulder was depressed, the hand elevated via a fulcrum at mid-forearm. The orthosis allowed a person to move horizontally, for example over a lap tray, and to use compensatory movements to attain limited movement in the vertical direction.

An enhanced version of the Balanced Forearm Orthosis allowed vertical movement by providing a horizontal joint at the base. The weight of the arm was compensated by means of rubber bands. In 1975, Burke Rehabilitation Centre modified the orthosis by adding 
actuators (dc motors). Control was maintained through use of a joystick, control pad, or various micro-switch assemblies and it provided $5 \mathrm{DOF}$ to the system.

\subsection{HYBRID ARM ORTHOSIS}

Hybrid Arm Orthosis (HAO) was developed by Benjuya and Kenney in 1990 (Benjuya \& Kenney, 1990). It was targeted for patients who are wheelchair bound due to postpolyomyelitis, high-level spinal cord injury or stroke. The purpose of the HAO was to restore hand/arm functions.

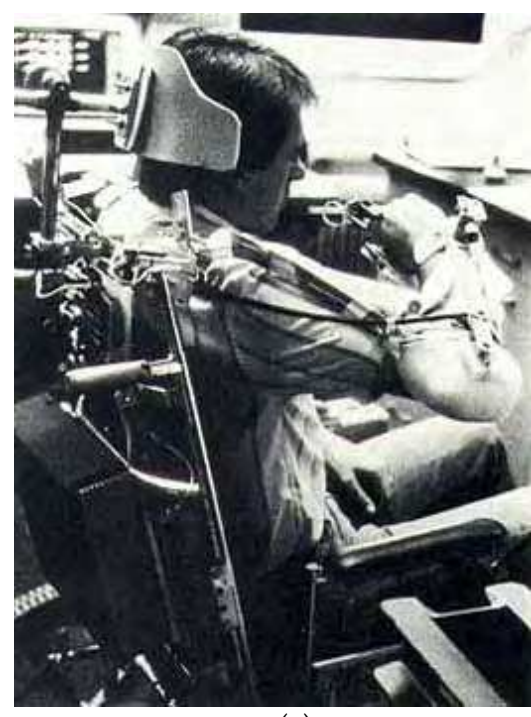

(a)

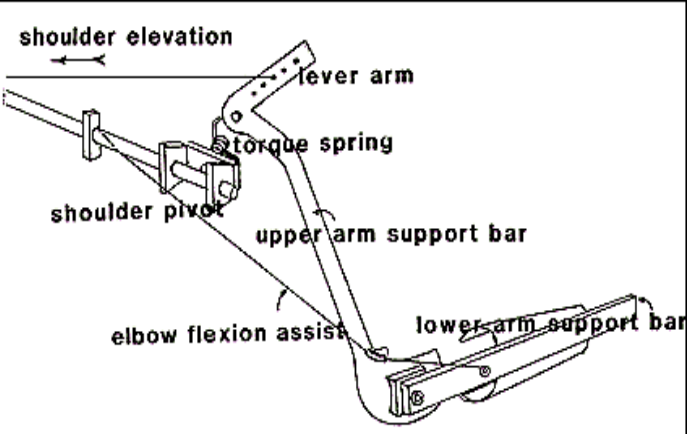

(b)

Fig. 2.6. Hybrid Arm Orthosis. (a) shows a patient wearing the exoskeleton (b) depicts the orthosis structure (Benjuya \& Kenney, 1990).

HAO achieved two major functions by using two different power sources. The shoulder and elbow joints were interconnected and simultaneously abduct and flex, respectively, by contra lateral shoulder elevation. The wrist supination and three-point jaw chuck pinch was generated by two separate switchable DC motors in sequence. In order to activate the motors, the patient slightly pressed air-switches that were located on the headrest of the wheelchair.

Clinical evaluation was limited to quadriplegics of C3-4 level. The patients tested dailyliving activities, such as self-feeding, with relative ease after minimal training (1-2 hours). In developing the HAO the feasibility and ease of modifying parts of the system for individuals with different needs was tested with success.

\section{Hand Exoskeletons}

Successful impairment diagnosis and continuous monitoring of progress are two important parameters of effective hand rehabilitation. Manual measurement of hand parameters is a timely and subjective task prone to errors. Sensing gloves could be a potential tool for increasing the efficiency and reliability of performing a rehabilitation task. Sensing gloves 
seem to be very effective in diagnosing hand disability because they provide excellent measurement of hand parameters and they don't require the presence of a medical specialist. Initial evaluation of a patient's impairment can be performed, which can be, accurately, repeatedly and objectively compared to follow up measurements.

Even though sensing gloves have been applied to hand diagnosis and rehabilitation they are unable, on their own, to apply resistive or assistive forces on the patient's hand. Forced mobilisation of joints is usually a necessary part of the treatment process. Researchers are addressing this with the application of robotic exoskeletons previously applied in Virtual Reality and Master-Slave telerobotics. Hand exoskeletons in particular have been in the centre of research due to their ability to apply forces to individual fingers and in some cases to multiple finger joints. Their multiple degrees of freedom offer increased quality and dexterity in finger joint mobilisation resulting to better therapy.

A review of the most relevant hand rehabilitation systems that have contributed by demonstrating a blend of technological advances and clinical outcomes is presented.

\subsection{VPL DATAGLOVE AND THE MOVEMENT ANALYSIS SYSTEM}

The first sensing glove to be applied to hand diagnosis was the DataGlove, developed by VPL. This was a thin lycra glove employing optic fibres as sensor elements for the measurement of finger positions figure 3.1. In its standard version it utilised two optic fibres per finger for the measurement of flexion and extension of the metacarpal and proximal interphalangeal joints. The principle of operation was measurement of light intensity attenuation as light travels through the optic fibre. The optic fibre's cladding was treated at the position of the measured finger joint so that its refractive index in combination with that of the core material allowed attenuation of light when the structure was bent. Therefore flexion of a joint can be measured by means of light intensity attenuation in the respective optic fibre.

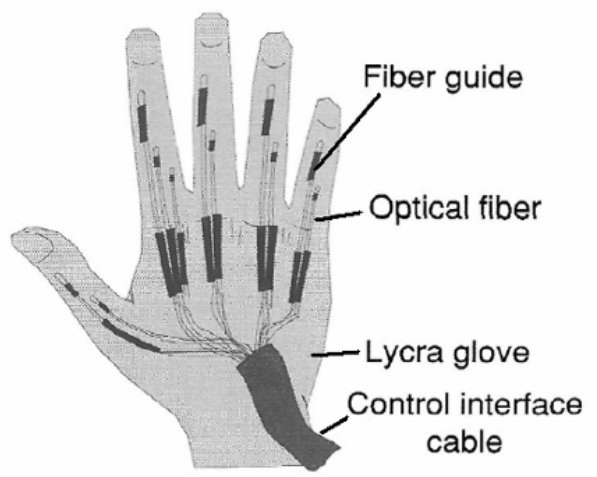

Fig. 3.1 The first version of the VPL DataGlove. Adapted from Burdea \& Coiffet, 1994.

Greenleaf Medical Systems was first to incorporate a sensing glove into a commercial hand diagnostic system (Greenleaf, 1992). This system called Movement Analysis System adapted the DataGlove's fibre optic technology and linked it with new software to create a tool for quantitative assessment of upper-extremity function. 


\subsection{CYBERGLOVE AND THE RUTGERS MASTER EXOSKELETON}

The Human-Machine Interface Laboratory at Rutgers has been a pioneer in the development of force feedback interfaces and was one of the first to develop a light weight portable hand exoskeleton (Burdea et al., 1992 b). This exoskeleton called Rutgers Master I (RMI) was the first to demonstrate high portability combined with relatively high force output. In 1992 Burdea and his colleagues first proposed the idea of a system for both diagnostic and rehabilitation of the hand (Burdea et al., 1992 a). The Rutgers Master II New Design (RMII-ND) is the latest version based on the RMII exoskeleton device that applies forces to the user's fingertips figure 3.2. It uses non-contact position sensors to measure the fingertip position in relation to the palm. Lightweight custom pneumatic actuators are attached to the tips of the thumb, index, middle, and ring fingers with force output up to $16 \mathrm{~N}$ (Bouzit et al., 2002).

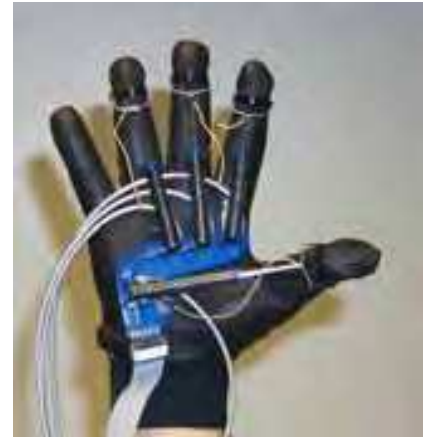

(a)

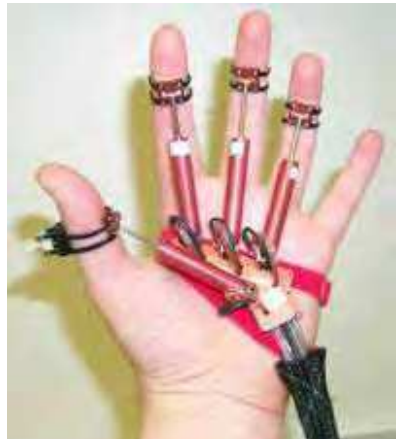

(b)

Fig. 3.2. (a) The Rutgers Master II force feedback glove, (b) The Rutgers Master II-New Design glove. Copyright Rutgers University.

In 2001 Jack et al proposed a hand rehabilitation system for stroke survivors (Jack et al., 2001; Boian et al., 2002; Adamovich et al., 2005). The aim of the proposed system was to offer dedicated exercises for finger range of motion, speed, fractionation and strength. The proposed system employs two devices to perform the aforementioned exercises. A Rutgers Master II-ND force feedback glove is used for exercises concerning finger strength, while a CyberGlove is used for the finger range, speed and fractionation exercises.

The Cyberglove is a sensing glove produced by the immersion corporation and is currently accepted to be the industry standard in hand tracking figure 3.3. The CyberGlove uses custom resistive bend sensors to measure the deflection of each finger. The 18-sensor model features two bend sensors on each finger, four abduction sensors, plus sensors measuring thumb crossover, palm arch, wrist flexion and wrist abduction.

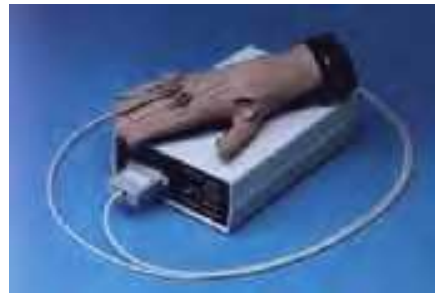

(a)

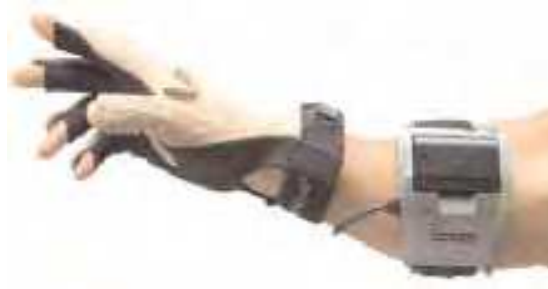

(b)

Fig. 3.3. The Cyberglove sensing Glove (a) Desktop version (b) Bluetooth wireless version. Picture Immersion Corporation. 
Treatment with this system is facilitated in a VR environment with game like tasks. Also web interconnectivity with patient progress databases, treatment parameter updates, video conferencing for telerehabilitation and online synchronous monitoring of multiple patients are some of the developed features. Automatic report generation of patient progress with detailed graphs has been also a part of this research.

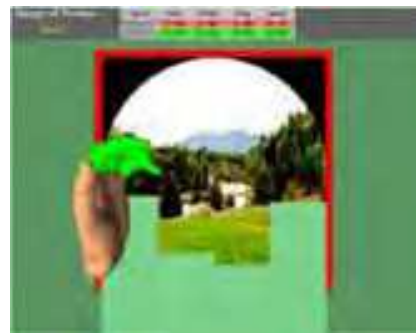

(a)

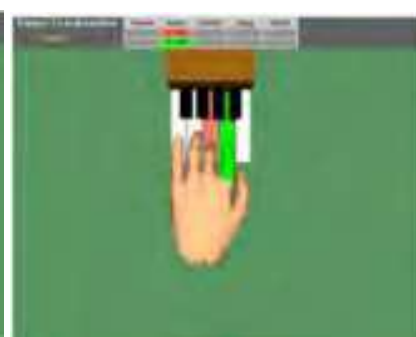

(b)

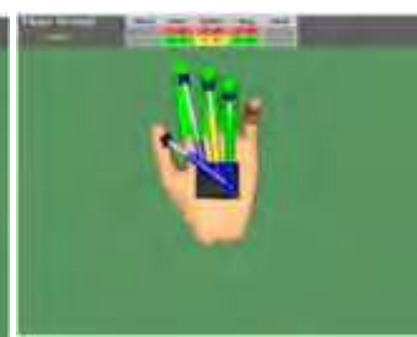

(c)

Fig. 3.4: Screen snapshots for the four VR exercises for post-stroke rehabilitation. (a)Range of Motion (b)Finger fractionation (c)Finger strength. Adapted from (Boian et al., 2002).

The four exercises were designed with World toolkit software development package. For the range-of-motion exercise, the patients have to flex their fingers to reveal pleasing images figure 3.4(a). In the speed exercise, the patients try to "chase away" a butterfly in the virtual scene by quickly flexing their fingers or thumb. In the fractionation exercise, the patients play a virtual piano keyboard pressing one key at a time figure $3.4(\mathrm{~b})$. For the strengthening exercise, which is performed with the RMII-ND the patient has to push down a piston with the thumb, index, middle, and ring fingers against a constant force figure 3.4(c). During exercise the patients receive auditory, visual, and numerical feedback about their target goal and their current performance. The target goals of the VR exercises are calculated automatically by the system, based on the patient's previous results (Adamovich et al., 2005).

To determine whether the skills gained in the VR environment transferred to real-world movements, two generalization tests were utilized, a clinical evaluation using the Jebsen Test of Hand Function and kinematic analysis of prehension movements. On average, the task was performed $22 \%$ faster after the intervention, illustrating transfer of their improvement in VR to a functional task (Adamovich et al 2005).

\subsection{P5 GAME GLOVE}

Rehabilitation and diagnostic systems based on high fidelity VR equipment like the DataGlove and Cyber glove are expensive and their cost may not be justified for general health care practice. In an effort to minimise this cost and to make VR therapy attractive for a more generalised use, (Morrow et al., 2006) proposed a system built around commercial video gaming equipment.

After successfully testing VR rehabilitation using high-end equipment in a clinical environment (Adamovich et al., 2005), the Rutgers group proceeded into applying the same therapeutic principles and exercises via alternative, more economical hardware. The CyberGlove was replaced by a P5 game glove while the PC formally responsible for graphics processing was substituted by an Xbox game console. The P5 game glove is a 5 DOF finger tracking glove which also incorporates $6 \mathrm{DOF}$ hand tracking. It employs one 
bend sensor on the dorsal side of each finger for flexion tracking with a resolution of $3^{\circ}$. With this configuration tracking of individual joint flexion is not performed rather a single value representing the total flexion is obtained. Hand tracking is performed by means of an optical infrared tracker. In this application tracking was limited to finger flexion while hand position and orientation in space was not implemented.

The aim of this project was to replicate the same finger velocity and range of motion exercises previously applied through a superior and more expensive system (Adamovich et al., 2005). Java 3D simulations were chosen to emulate the exercises initially developed with WorldToolKit graphics development platform alleviating the high cost of the latter. Also extensive interventions on both Xbox's hardware and software have been performed in order to allow interconnection of hardware and compatibility with the Java 3D VR application. Figure 3.5 presents the system components.

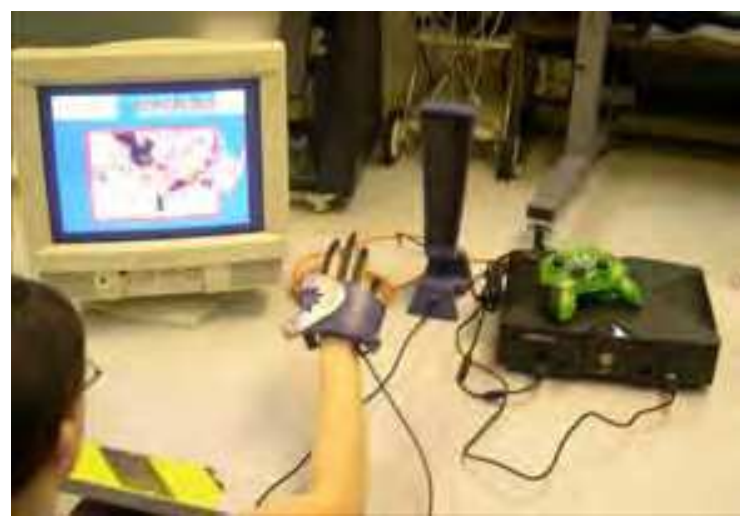

Fig. 3.5. Overall view of the experimental low-cost finger training system. Adapted from (Morrow et al., 2006).

According to the researchers this approach had been proven to be highly cost effective since it produced a system costing only $\$ 549,32$ times cheaper than its precursor. Nevertheless, overall business running costs for the adaptation of a bulk of such systems have not been calculated and thus a final price figure remains to be seen. As expected the claimed cost savings come at a trade-off in functionality and accuracy since CyberGlove is definitely offering superior performance to the gaming P5 Glove. If further clinical testing proves that such a system is beneficial to post-stroke hand rehabilitation, it will provide an answer to the cost related scepticism towards VR and tele-rehabilitation systems.

\subsection{HAND WRIST ASSISTING ROBOTIC DEVICE (HWARD)}

Recent encouraging findings of research on motor retraining following a cerebrovascular accident have motivated researchers at the University of California to develop a hand assisting robotic device for stroke rehabilitation (Cramer et al., 2007). The system called HWARD (Hand Wrist Assisting Robotic Device) is a 3 DoF device that exercises flexion and extension of the hand as well as some wrist movement. The aim was to retrain hand grasping and releasing movements while simultaneously using real objects during therapy. This is achieved by providing an unobstructed palm area where various objects can be offered for interaction during exercise. In this manner assisted grasping and releasing of objects can be combined with tactile stimuli, which is useful for associating and retraining tactile sensation in grasping. 
HWARD is a pneumatic actuated desk mounted exoskeleton that supports the patients arm and is attached on the thumb and fingers as shown in figure 3.6. The device can flex or extend all 4 fingers together about the metacarpophalangeal (MCP) joint, the thumb at the $\mathrm{MCP}$ joint, and the wrist. Joint angle sensors in the structure are used to measure the movement of the exoskeleton's joints, and hence, movement of the subject's limbs when attached to the device.

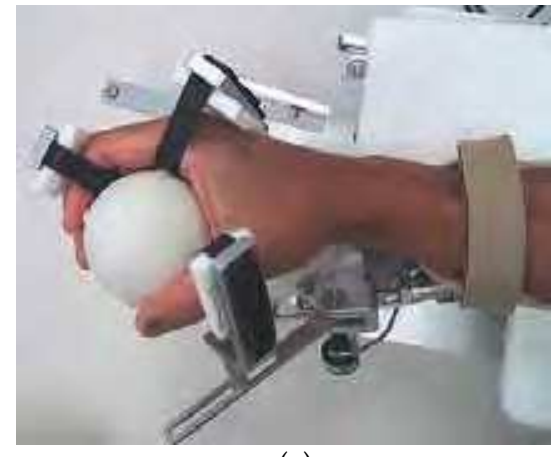

(a)

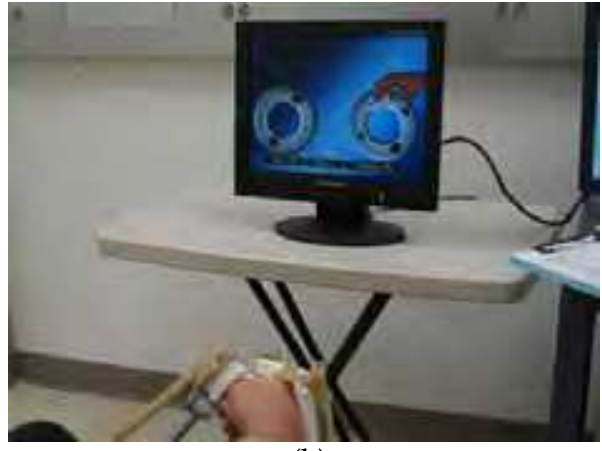

(b)

Fig. 3.6. HOWARD hand exoskeleton. (a)The exoskeleton allows practice of grasping with real objects; (b) practice of grasping in a VR environment. Adapted form (Cramer et al 2007).

A therapy program based on this device was developed and emphasised right hand movement speed, force, precision, timing, and repetition, and included virtual reality/games.

In a pilot study to assess the efficacy of the system, a selection of thirteen patients of an average age of 63 received 15 two-hour therapy sessions spread over three weeks. During treatment all subjects worked with HWARD. The sessions were a mixture of grasp/release protocols of real/virtual objects. Seven of the subjects received full support from the system throughout each session. For the remaining six patients full assistance in the grasping and releasing exercises was offered only in the second part of each session.

To objectively measure the effect of therapy all patients were assessed prior and post treatment using three tests. These tests were Action Research Arm Test, Box-and-Blocks Test and the standard occupational therapy assessment tool called the Fugl-Meyer score. At the end of treatment the results showed an average improvement of 10 and 20 percent in the first two tests respectively, which assess occupational functionality and dexterity, while they were all rated as less disabled according to the Fugl-Meyer score. An increase of 17 percent in the range of motion was also observed. Comparing the average gains of the two groups of patients, in the three assessment tests, researchers observed a twofold increase, in all test, in the group that had received full assistance throughout each session. These changes in functionality within each subject before and after the three week treatment were assessed as highly significant. An interesting finding of this study supported by functional MRI tests was that functional gains obtained for practised motions did not extend to unpractised motions. This close association of gain and practiced task indicates the need for both intensive and diverse VR therapy in order for a generalised functional improvement. 


\subsection{THE HAND MENTOR}

The hand mentor is the first commercial hand rehabilitation Active Repetitive Motion ${ }^{T M}$ therapy system produced by Columbia Scientific LLC. The hand Mentor is a single degree of freedom device that provides a controlled resistive force to the hand and wrist figure3.7. The applied force can oppose flexion or assist extension of the hand. It incorporates sensors that monitor the position of wrist and fingers during flexion-extension motions as well as force sensors to measure the force applied on the hand by the system actuator.

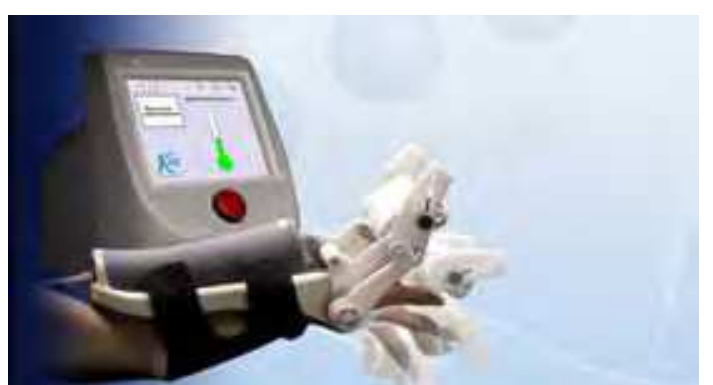

Fig. 3.7. Hand Mentor, the first commercial Active Repetitive Motion ${ }^{\mathrm{TM}}$ hand therapy device. Adapted from Columbia Scientific LLC.

The actuator is a compliant air muscle actuator with a combination of high power and high compliance (Caldwell et al., 1994). The device incorporates surface electromyography (EMG) recording electrodes in contact with the patient's muscles and an EMG level display. In an exercise with the Hand Mentor the patient initially works towards a target without any assistance from the device while observing the EMG indication display. Observation of the EMG level display provides a reliable feedback of muscle firing that can gradually train the patient to intuitively achieve muscle coordination. When the patient reaches the limit of his motion range the device actively assists the motion. Stretching beyond this limit helps to reduce spasticity and muscle tone. In order to investigate the potential of the hand mentor as a clinical tool for hand rehabilitation post stroke, a clinical study was carried out (Kinetic Muscles Inc. 2005). The study protocol consisted of eight patients attending sessions five days a week for three weeks for 3 hours a day. The device was used for 1.5 hours each day and 1.5 hours were spent on repetitive task practice with a clinician. The assessed hypotheses were weather Mentor could work in a clinical study, the effectiveness of Active Repetitive Motion Therapy in restoration of hand function, and the cost effectiveness of such a system.

The Mentor was well accepted by patients. The therapy was assessed to work in a clinical setting. ARM therapy was effective in restoring function in the selected sample of stroke patients. By using therapist time more efficiently, the therapy seemed to be cost-effective compared to conventional CI therapy. This device's portability and reasonable cost could allow for a lending regime for home therapy where the patient can exercise intensively in his own time at the convenience of his home. This would reduce travel cost and expensive clinic time.

\section{Salford Rehabilitation Exoskeleton}

Tsagarakis et al at the University of Salford (Tsagarakis et al, 2003) have designed and built a multi-jointed gravity compensated upper arm assistive exoskeleton. The use of novel pneumatic actuation techniques (Caldwell et al., 1994) provides a design with accurate 
position and forced controlled paths, compliance and a high level of inherent safety that is capable of controlled path and force trajectories in a complex 3D workspace. SRE's mechanical design, figure 4.1, has 7 degrees of freedom (DOF). Three of these DOF are located at the shoulder permitting flexion/extension, abduction/adduction and lateral/medial rotation. Two are located at the elbow permitting flexion/extension and pronation/supination of the forearm. The remainders are located at the wrist permitting flexion/extension and abduction/adduction. More details about the mechanical structure can be found here (Tsagarakis \& Caldwell, 2003).

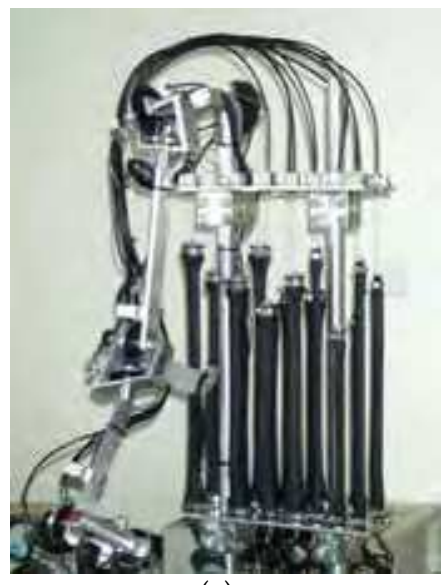

(a)

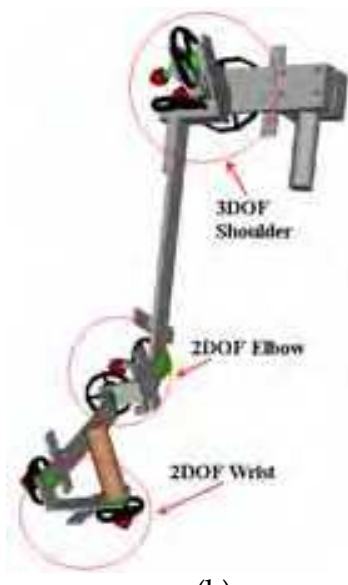

(b)

Fig. 4.1. Salford Rehabilitation Exoskeleton. (a) The arm exoskeleton; (b) 3Dimensional model of the exoskeleton structure (Tsagarakis et al., 2003).

The exoskeleton framework is light due to its fabrication in aluminium with stressed components in steel (approx. weight $2 \mathrm{~kg}$ ) although the use of gravity compensation means that a user does not need to support any load if this is required. It is attached to the user at the elbow via a Velcro strip which makes it comfortable to wear, easily fitted and more acceptable to the patients. The workspace of the system permits motion over $75 \%$ of the volume of normal operation (Tsagarakis \& Caldwell, 2003) permitting excellent duplication of the motions needed in completion of real world tasks. Dedicated software permits the control of the exoskeleton in three different modes: joint position control, joint torque control and impedance control respectively (Tsagarakis \& Caldwell, 2003).

Rehab Lab is a computer generated therapy environment (Kousidou et al., 2006) that supports task-based therapy and through which, therapists can synthesise therapeutic protocols. In order to synthesise a protocol, a therapist combines a number of tasks with information about the number of repetitions, the interval after each repetition and the resting period between the different tasks. The tasks can vary from simple (i.e. shoulder flexion/extension) to more complicated (e.g. reaching tasks). Rehab Lab enables SRE to operate in three modes. These modes vary from full assistance from the exoskeleton to no assistance (recording and monitoring only), according to the recovery stage the patient is in. The modes are: Full Assistive mode (FA), Partial Assistive mode (PA), Non Assistive mode (NA). 
During the first stages of recovery and while the patient cannot move his/her limbs without assistance, the full assistive mode can be used in order for the protocols to be executed with full assistance from the exoskeleton. All protocol tasks are executed at a constant speed (which can be adjusted for different tasks). This mode extends the concept of isokinetic machines already used in rehabilitation and has been evaluated in (Kousidou et al., 2003). While isokinetic machines offer only single joint exercises, Rehab Lab can exercise multiple joints simultaneously. Start and stop angles as well as speed, are the necessary parameters for trajectory generation of single joint exercises. For multi-joint exercises, trajectories are produced by recording a motion using the exoskeleton and then playing it back. Partial assistive mode is implemented with the help of a force/torque $(\mathrm{F} / \mathrm{T})$ sensor attached to the wrist of the exoskeleton. The sensor detects intention of movement (through a sensitivity scaling of the sensor's values) and Rehab Lab moves SRE accordingly. In non assistive mode, the exoskeleton is configured to simulate the forces generated by an exercise. This mode of therapy can be used when the patient has regained enough strength to complete a protocol on his/her own. In this mode, an impedance controller accepts as input the torques produced by the user and the control mode changes from position to impedance control.

Rehab Lab also contains a Virtual Environment (VE) figure 4.2. The VE provides the setup for the tasks to be performed but can also be manifested as a means of biofeedback. Currently, only one set-up exists which contains a table and a number of objects placed on its surface. After the therapist selects the suitable protocol for the patient, he/she can preview it in the VE. A female avatar is demonstrating the protocol so that the patient has a better idea of the sub-tasks to be performed. Once instructed in the protocol, the exoskeleton is fitted to the patient and the patient is asked to complete the tasks.

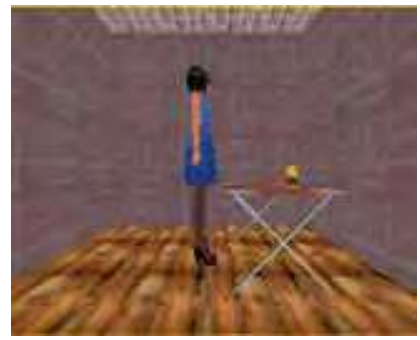

Fig. 4.2. The VE in Rehab Lab. The figure depicts a female avatar getting ready to perform a reaching task.

During the protocol, the patient can see a reflection of his/her movements in the virtual environment. That is, a virtual character is sitting in exactly the same position as the patient and his arm is moving exactly the same way as the patient's. The virtual character's arm position is updated by the exoskeleton's position sensors. This awareness helps them establish the boundaries of their limb with respect to the environment and the objects they have to manipulate and therefore learn how to control their responses.

In a pilot study involving healthy subjects, reaching tasks were performed (with no, partial and full assistance form the exoskeleton) in order to test the system's ability to replicate complex input trajectories. The results were encouraging as position sensing showed that output trajectories for reaching tasks were well correlated with inputs to the exoskeleton. EMG evaluation demonstrated the successful switching between the various modes of exercise. Results for a typical reaching task are shown in figure 4.3 and figure 4.4. 


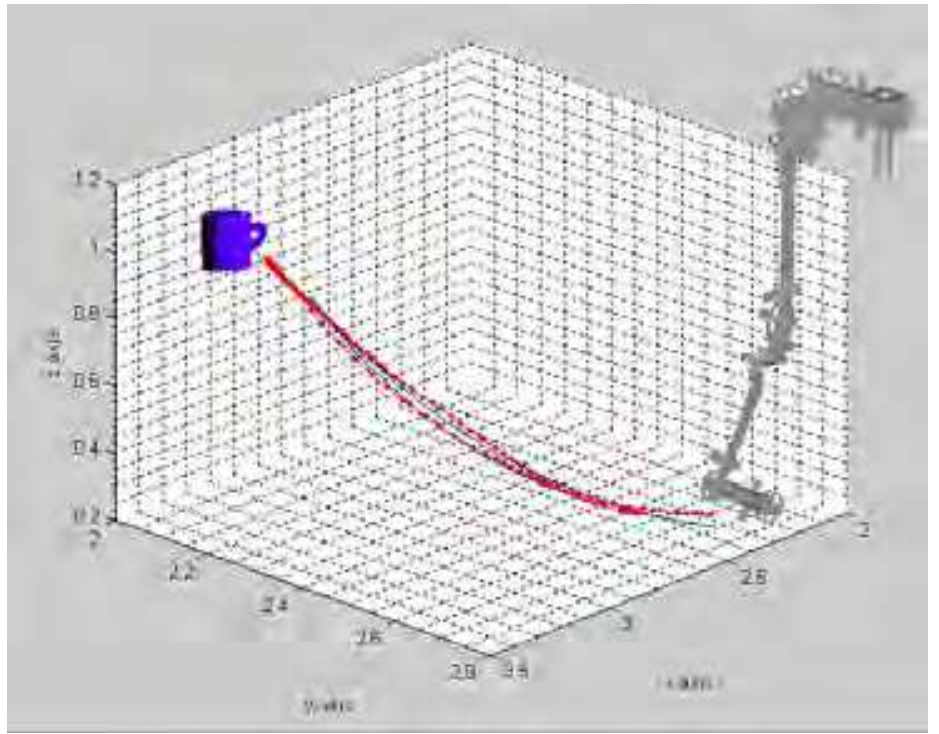

Fig. 4.3. Typical reaching task. The thin blue line denotes the input trajectory and the red thick line the actual exoskeleton trajectory as the task was performed. As seen, the red line follows the blue line with a high degree of correlation. (Kousidou et al., 2006)

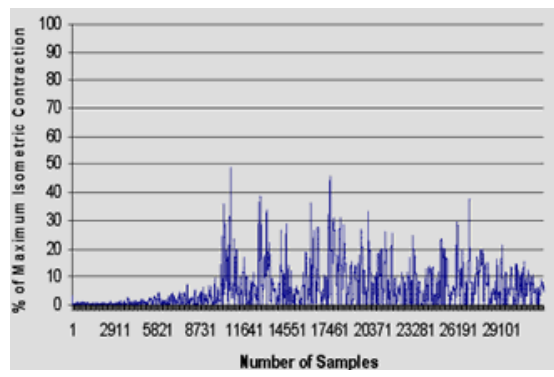

(a)

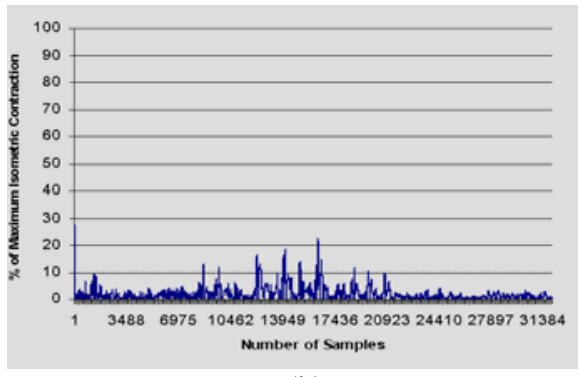

(b)

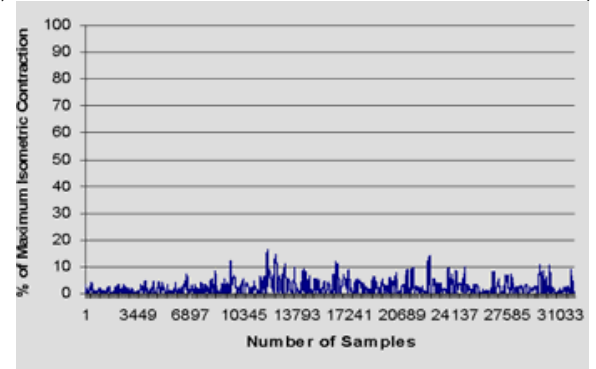

(c)

Fig. 4.4. Typical reaching task. (a) is a typical muscle activity snapshot during non-assistive mode; (b) during a partial assistive mode and (c) during full assistive mode. (Kousidou et al., 2006) 


\section{Salford University Exoskeleton Hand Exerciser}

A common problem of the presented hand exoskeletons is their inadequacy to combine different modalities such as hand parameter diagnosis and exercise assortment with qualities such as finger dexterity and satisfactory finger range of motion. Sarakoglou et al (Sarakoglou et al., 2004) have proposed an exoskeleton exerciser that combines dexterity with a good range of motion. The system enables the execution of finger therapy regimes and can also be used as a motion analysis and lost finger mobility diagnosis tool. The overall aim of this system is to provide physiotherapy regimes in an interactive virtual environment using a hand exoskeleton based exerciser.

As far as the therapeutic functionality of this system is concerned it provides facilities for hand motion tracking, recording and analysis as well as ability of execution of both occupational and physical therapy exercises.

\subsection{Mechanical Design}

The mechanical structure of the hand exerciser provides 7 active degrees of freedom. The Exoskeleton resides on the dorsal side of the hand and the forces are applied from that direction. The forces are generated by dc motors mounted in a low profile power pack and are transmitted to the fingers by low friction tendons. Finger force reflection that is generated by DC motors is accurately controlled by means of strain gauge based sensors. Measurement of the finger flexion is achieved by a combination of flexible resistive sensors integrated in a soft lycra glove and custom made linear electromagnetic sensors embedded in the exoskeleton's metallic structure. The incorporated glove unlike other systems is part of the exoskeleton structure and thus it is faster to put on and take off. The design also allows for fast adjustment of the exoskeleton for different hand sizes. Patient safety is provided by mechanical stops that limit the finger motion to within acceptable range in case of exoskeleton loss of control.

This exoskeleton is a single wearable device although it can be visualised as 3 main modules, figure 5.1. These are: 1) The exoskeleton, that consists of the hand support plate and the aluminium structure that helps to transmit the feedback forces to the finger joints, 2 ) the glove unit and 3) the Power pack.

The developed exoskeleton can apply forces to the index, middle and ring fingers and to the thumb. The applied forces can either resist flexion or assist extension of the fingers. For the first three fingers force is provided to proximal and distal phalanges while for the thumb only the distal phalanx is currently active. The exoskeleton is designed to fit a range of hand sizes and for this purpose it incorporates adjustment levers that allow fast and easy adjustment of the metallic structure for the three fingers. A 3D Cad drawing of one finger is presented in Figure 5.2.

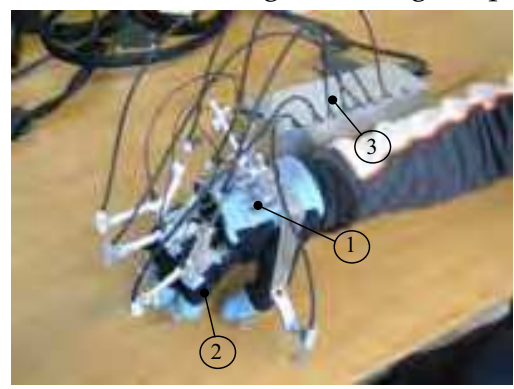

Fig. 5.1. The hand exoskeleton indicating the three basic modules. Adapted from (Sarakoglou et al., 2004). 
To address the requirement for accurate finger tracking the hand exoskeleton employs a combination of input sensors. As mentioned previously it incorporates a lycra glove, which facilitates the housing of seven resistive flex sensors that measure finger flexion. Four flex sensors measure the flexion angle of the middle joints of the three fingers. The angles of the distal inter-phalangeal joints $\theta_{3}$ (equation (1)) of the fingers are calculated from the middle joint angle $\theta_{2}$ which is directly measured by a flex sensor (Gomez et al., 1995). Another three sensors monitor the flexion, rotation, abduction and adduction of the thumb.

$$
\theta_{3}=0.46 \cdot \theta_{2}+0.083 \cdot \theta_{2}^{2}
$$

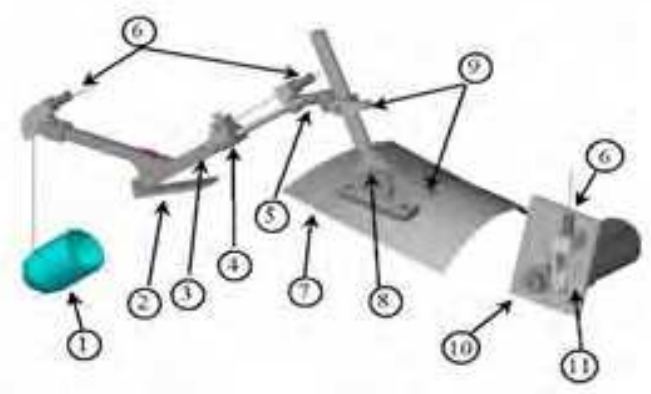

Fig. 5.2. Main components of the exoskeleton: 1-Finger Cup, 2-metalic plate attached with Velcro to the proximal phalanx,3-Structure for support and finger flexion measurement, 4Steel rod \& linear bearing, 5-Universal Joint, 6-Pull cables for force transmission, 7Cushioned thermoplastic plate for exoskeleton support on the hand, 8-Adjustable Support, 9-Adjustment levers, 10-Motor module, 11-Strain gauge for measurement of force applied to the fingers. Adapted from (Sarakoglou et al., 2004).

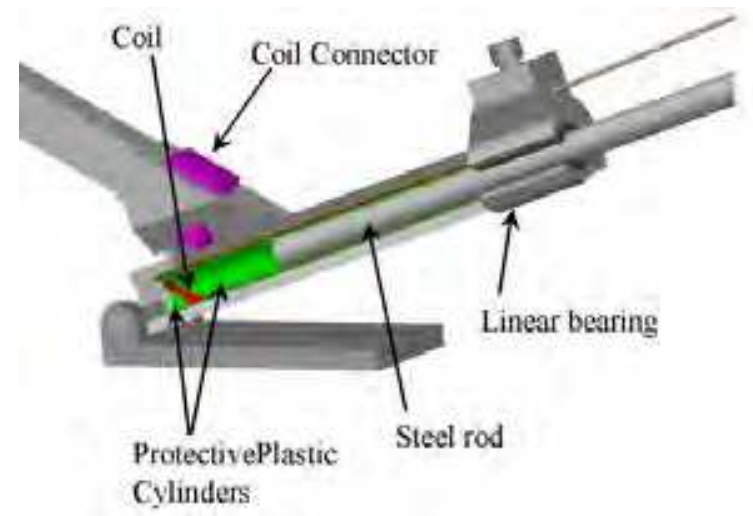

Fig. 5.3. Linear electromagnetic sensor integrated in the metallic structure. Adapted from (Sarakoglou et al., 2004)

Because of the obstruction posed by the mechanical structure placed on the back of the hand and on the proximal phalanges, the flexion of the proximal joint is not performed with flex sensors. Instead a custom made linear electromagnetic sensor is employed that is embedded in the metallic structure, figure 5.3. 


\subsection{System Integration and Therapeutic Environment}

To enable the execution of hand therapy exercises the system was integrated within a Hand Therapy System. This therapy system, which is shown in figure 5.4 formed the test-bed where a clinician can customise and perform both finger motion evaluation tests and hand therapy regimes. The later resemble real tasks as much as possible particularly in terms of mechanical movements.

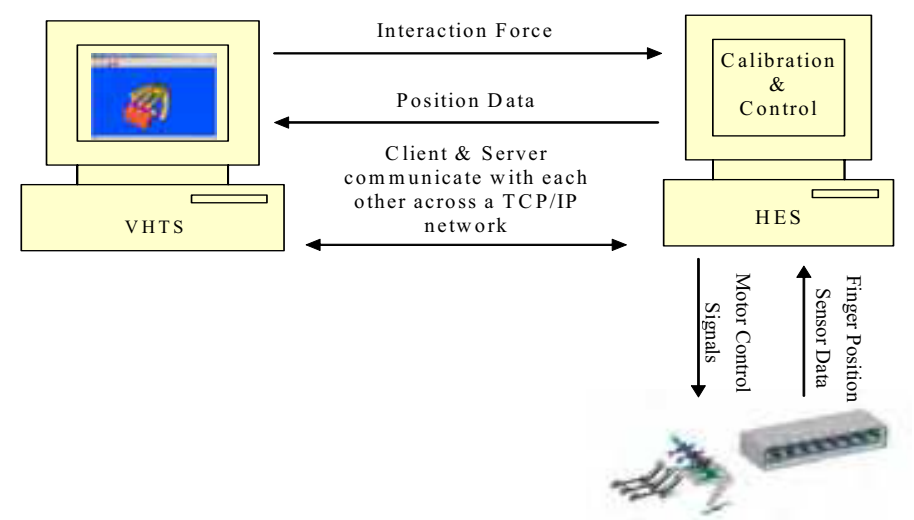

Fig. 5.4: Hand Therapy System Configuration. Adapted from (Sarakoglou et al., 2004).

The Hand therapy system consists of the Virtual Hand Therapy Station (VHTS) and the Hand exerciser Server (HES). The VHTS consists of a dedicated graphics machine that is responsible for the execution of the software relating to the exercise customisation and visualization. The HES is realized using a dedicated PC, which executes the software modules associated with the Hand Exerciser device calibration and control. Communication between the (VHTS) and (HES) is performed using a dedicate TCP/IP link. The aim of the Virtual Hand Therapy system is to perform hand rehabilitation using simple tasks. Since the exact type of deficiency can vary tremendously from patient to patient, even with the same diagnosis, the system was designed to be flexible so that the training can be adjusted to the needs of different patients. The virtual exercises resemble the real tasks as much as possible with the care that very complex, realistic or fanciful graphics and fastpaced game formats may be overwhelming for patients. In contrast, a very simple display, with few movements may help a patient to focus on the task at hand and enhance learning.

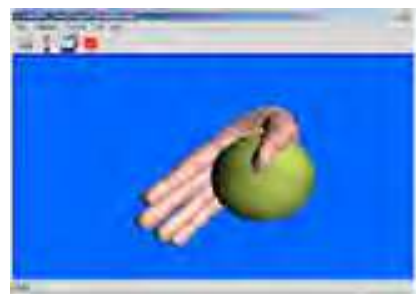

(a)

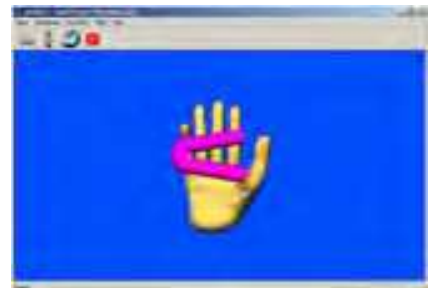

(b)

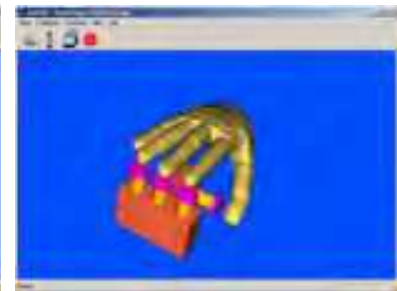

(c)

Fig. 5.5. Implemented VR Exercises: (a) Simulation of elastic ball exerciser, (b) Simulation of hand grip exerciser, (c) Simulation of trumpet keys exerciser (fractionation exercise). Adapted from (Sarakoglou et al., 2004). 
In this respect existing therapy regimes constitute the basic principles in designing the VR physical exercises. Based on these existing physiotherapy methods recommended by physicians, three initial exercise regimes were developed. Figure 5.5(a) and figure 5.5(b) present VR Exercises simulating the action of existing manual exercisers. The aim of these exercises is to strengthen grip and increase hand motion. In the third exercise figure 5.5(c) the patient is required to push down the virtual model of a trumpet key. In order to do so, the patient must resist the opposing force of the virtual spring in the piston and flex his fingers. The stiffness of the VR objects is increased as the patient's therapy progresses.

A number of experimental trials were carried out to evaluate the performance of the system. Although the tests were performed by healthy subjects, they indicated that the system is reliable enough to undergo further clinical trial.

\section{Discussion and conclusions}

In this chapter we have stated the need for power-assisted and robotic tools as means of providing intensive, cost-effective and objectively measured physiotherapy. We presented the state-of-the-art as well as early exoskeleton systems targeted at upper arm and hand disabilities. We also presented work that is undergoing at the University of Salford, UK, an upper arm and a hand exoskeleton that can be used as potential tools for delivering devicemediated rehabilitation. Clearly, device-mediated rehabilitation is an active area of research whose findings have great impact on our society's well-being.

One could argue that the systems reviewed in the previous sections define or have already defined, in one way or another, future trends in rehabilitation robotics. Based on the advantages and drawbacks of the systems presented, we can draw some rough design and functionality requirements of an effective rehabilitation system.

The number of degrees of freedom is important for a rehabilitation facility as it allows for a broad range of tasks to be performed. Exoskeletons are expected to play a big role in the future of physical and occupational therapy as they offer a more natural way of interaction with real or virtual environments. The latter happens due to the fact that they utilise most of the human physical workspace. The weight and the volume of the system are also important. Adequate force/torque output, adequate range of motion and accurate position tracking are also required for such systems. Safety mechanisms at mechanical, electrical and software level need to be incorporated in order for a system to become commercial.

Virtual environments are also increasingly considered as a key component in rehabilitation: they can provide visual and auditory interactions and when used in conjunction with haptic feedback, they can engage a patients' attention while at the same time keeping them motivated. VEs also provide therapists with a set-up for repetitive functional ADL training or any other form of intensive training while at the same time giving quality feedback to patients helping them control their physiological responses in an engaging and entertaining way. EMG measurements can monitor levels of muscle activity and therefore can be included in a rehabilitation system as an indicator of recovery levels.

What is clearly missing from the prior art though, is a standard method of delivering therapy consisting of a unified way of synthesising therapeutic protocols and which could potentially be used as a common platform by researchers and therapists. A common platform such as this could promote a better insight into the nature of disability treatment as it would create the opportunity for keeping a certain number of variables constant (such as exercise type and parameters) while exploring others. 
Device-mediated rehabilitation is an active area of research whose findings have great impact on our society's well-being. There are many problems that need to be solved but the cause of producing effective rehabilitation systems is very important and therefore worth persisting upon.

\section{References}

Adamovich S., Merians, A., Boian, R., Tremaine, M., Burdea, G., Recce, M., \& Poizner, H. (2005). A Virtual Reality Based Exercise System for Hand Rehabilitation PostStroke. Presence, Special Issue on Virtual Rehabilitation, Vol.14, No.2, April 2005 pp.161-174, ISSN:1054-7460

Alexander, M.A.; Nelson, M.R. \& Shah, A. (1992). Orthotics, Adapted Seating and Assistive Devices, Pediatric Rehabilitation 2nd Edition, pp. 186-187, Baltimore MD, 1992, Williams and Wilkins (eds)

Benjuya, N. \& Kenney, S.B. (1990). Hybric Arm Orthosis, Journal of Prosthetics and Orthotics, Vol. 2, No. 2 (2nd Quarter, 1990), pp. 155-163, ISSN 1040-8800

Boian, R., Sharma, A., Han, C., Merians, A. S., Burdea, G., Adamovich, S., Recce, M., Tremaine, M., \& Poizner, H. (2002). Virtual Reality-Based Post Stroke Rehabilitation, Proceedings of Medicine Meets Virtual Reality 2002, pp. 64-70, IOS Press, January 2002, Newport Beach CA.

Bouzit, M., Popescu, G., Burdea, G, \& Boian, R. (2002). The Rutgers Master II-ND Force Feedback Glove, Proceedings of VR 2002 Haptics Symposium, pp. 145-152, Orlando FL, March 2002, IEEE, Orlando FL

Burdea, G \& Coiffet, P. (1994). Virtual Reality Technology, 1st Ed, John Wiley \& Sons, Inc., ISBN 2-8666601-386-7, New York:

Burdea, G., Roskos, E., Silver, D., Stone, R., \& Dipaolo, D. (1992 a). Diagnostic / Rehabilitation System using Force Measuring and Force Feedback Dexterous Masters, Proccedings of Medicine Meets Virtual Reality 5 Conference, pp.18-10, June 1992, San Diego, CA

Burdea, G., Zhuang, J., Roskos, E., Silver, D., \& Langrana, N. (1992 b). A portable dextrous master with force feedback. Presence: Teleoperators and Virtual Environments Vol. 1, No. 1, (Jan. 1992),pp 18-28, ISSN1054-7460

Burgar, C.G.; Lum, P.S.; Shor, P.C. \& Van der Loos, M. (2000). Development of robots for rehabilitation therapy: the Palo Alto VA/Stanford experience, Journal of Rehabilitation Research and Development, Vol. 37, No. 6. (November/December 2000), pp. 663-73, ISSN 0748-7711

Caldwell, D.G.; Medrano-Cerda, G.A. \& Goodwin, M.J. (1994). Characteristics and adaptive control of pneumatic muscle actuators for a robotic elbow, Proceedings of IEEE International Conference on Robotics and Automation, pp. 3558-3563, US, May 1994, San Diego, IEEE, California

Carr J.H. \& Shepherd, R.B. (1987). A motor Relearning Programme for Stroke, Butterworth Heinemann, ISBN 0871893126, Oxford

Cozens, J.A. (1999). Robotic assistance of an active upper limb exercise in neurologically impaired patients, IEEE Transactions on Rehabilitation Engineering, Vol. 7, No.2 (June 1999), pp. 254 - 256, ISSN 1063-6528

Cramer, S.C., Takahashi, C.D., Der-Yeghiaian, L., See, j., Motiwala, R.R. \& Le, V. (2007) Robot-Based Hand Motor Therapy after Stroke, Presented in the International Stroke Conference, Feb. 2007, San Francisco, CA 
Gomez, D., G. Burdea \& N. Langrana. (1994). The Second Generation Rutgers Master - RM II, Proceedings of Automation'94 Conference, Vol. 5 pp.7-10, Taipei, July 1994. Taiwan

Greenleaf, W. J. (1993). DataGlove, DataSuit and Virtual Reality Advanced Technology for People with Disabilities, Proceedings of Virtual Reality and Persons with Disabilities. California State University, 1993, Northridge, CA

Hogan, N.; Krebs, H. I.; Charnnarong, J.; Srikrishna, P. \& Sharon, P. (1992). MIT-MANUS: A workstation for manual therapy and training, Proceedings of IEEE Workshop on Robot and Human Communication, pp. 161-165, 0-7803-0753-4, Japan, September 1992, IEEE, Tokyo

Jack, D., Boian, R., Merians, A. S., Tremaine, M., Burdea, G. C., Adamovich, S. V., Recce, M. \& Poizner, H. (2001). Virtual Reality-Enhanced Stroke Rehabilitation. IEEE Transactions on Neural Systems and Rehabilitation Engineering, Vol.9, No.3, September 2001, pp 308-318, ISSN:1534-4320

Johnson, G.R.; Carus, D.A.; Parrini, G.; Scattareggia Marchese, S. \& Valeggi, R. (2001). The design of a five-degree-of-freedom powered orthosis for the upper limb, Proceedings of the Institution of Mechanical Engineers Part H-Journal of Engineering in Medicine, Vol. 215, No. 3 (2001), pp. 276 - 284, ISSN 0954-4119

Kinetic muscles Inc. (2005). Active Repetitive Motion ${ }^{\mathrm{TM}}$ (ARM) Therapy for Increasing the Function and Independence of Stroke Patients,Report Submitted for NIH Grant Approval 2005

Kousidou, S.; Tsagarakis, N.G. \& Caldwell, D.G. (2003). Evaluation of a "soft" exoskeleton for rehabilitation and physiotherapy of the upper limb Proceedings of IEEE International Conference on Advanced Robotics, pp. 1080-1085 Portugal, June 2003, IEEE, Coimbra

Kousidou, S.; Tsagarakis, N.G. \& Caldwell, D.G. (2006). Assistive Exoskeleton for TaskBased Physiotherapy in 3-Dimensional Space, IEEE International Conference on Biorobotics and Biomechatronics, pp. 266- 271, Italy, February 2006, IEEE, Pisa

Mihelj, M.; Nef, T. \& Riener, R. (2006). ARMin - Toward a Six DoF Upper Limb Rehabilitation Robot, IEEE International Conference on Biorobotics and Biomechatronics, pp. 1154- 1159, Italy, February 2006, IEEE, Pisa

Morrow, K., Docan, C., Burdea, G. \& Merians, A. (2006). Low-cost Virtual Rehabilitation of the Hand for Patients Post-Stroke, Proceedings International Workshop on Virtual Rehabilitation, pp 6-10, ISBN: 1-4244-0280-8, August 2006, New York, NY

Nakayama, H.; Jorgensen, H.S; Raaschou, H.O. \& Olsen, T.S. (1994). Recovery of upper extremity function in stroke patients: the Copenhagen Stroke Study, Archives of Physical Medicine and Rehabilitation, Vol. 75, No.4 (April 1994), pp. 394-8, ISSN 0003-9993

Parker, V.M.; Wade, D.T. \& Langton, H.R. (1986). Loss of arm function after stroke: measurement, frequency, and recovery, International Rehabilitation Medicine, Vol. 8, No.2 (1986), pp. 69-73, ISSN 0379-0797

Rahman, T.; Sample, W.; Seliktar, R.; Alexander, M. \& Scavina, M. (2000). A Body-Powered Functional Upper Limb Orthosis, Journal of Rehabilitation Research and Development, Vol. 7, No. 6. (November/December 2000), pp. 675-680, ISSN 07487711 
Ruiz A.F.; Forner-Codrero, A.; Rocon, E. \& Pons, J.L. (2006). Exoskeleton for Rehabilitation and Motor Control, IEEE International Conference on Biorobotics and Biomechatronics, pp. 601- 606, Italy, February 2006, IEEE, Pisa

Sarakoglou, I., Tsagarakis, N.G. \& Caldwell, D.G. (2004). Occupational And Physical Therapy Using A Hand Exoskeleton Based Exerciser, Proceedings of Intelligent Robots and Systems, (IROS 2004), Vol: 3, pp 2973- 2978, ISBN: 0-7803-8463-6, Sept.Oct. 2004 IEEE/RSJ, Sendai, Japan

Tsagarakis, N.G. \& Caldwell, D.G. (2003). Development and control of a physiotherapy and training exercise facility for the upper limb using soft actuators, Proceedings of IEEE International Conference on Advanced Robotics, pp. 1092-1097, Portugal, June 2003, IEEE, Coimbra

Volpe, B.T.; Krebs, H.I.; Hogan, N., Edelstein, L.; Diels, C. \& Aisen, M. (2000) A novel approach to stroke rehabilitation: robot-aided sensorimotor stimulation, Neurology, Vol. 54, No. 10 (May 2000), pp. 1938-44, ISSN 0028-3878 


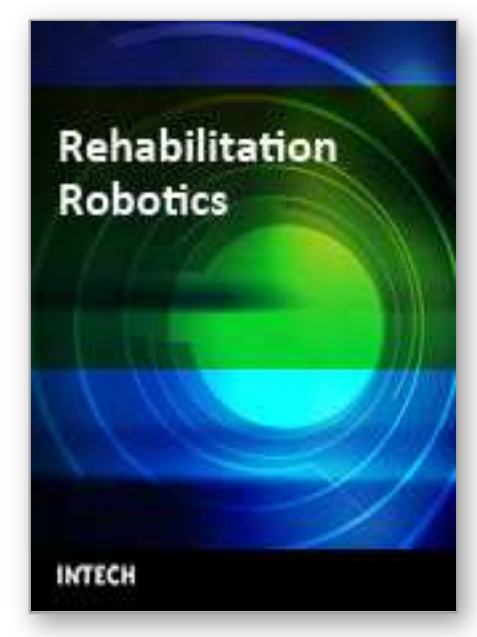

\author{
Rehabilitation Robotics \\ Edited by Sashi S Kommu
}

ISBN 978-3-902613-04-2

Hard cover, 648 pages

Publisher I-Tech Education and Publishing

Published online 01, August, 2007

Published in print edition August, 2007

The coupling of several areas of the medical field with recent advances in robotic systems has seen a paradigm shift in our approach to selected sectors of medical care, especially over the last decade.

Rehabilitation medicine is one such area. The development of advanced robotic systems has ushered with it an exponential number of trials and experiments aimed at optimising restoration of quality of life to those who are physically debilitated. Despite these developments, there remains a paucity in the presentation of these advances in the form of a comprehensive tool. This book was written to present the most recent advances in rehabilitation robotics known to date from the perspective of some of the leading experts in the field and presents an interesting array of developments put into 33 comprehensive chapters. The chapters are presented in a way that the reader will get a seamless impression of the current concepts of optimal modes of both experimental and ap- plicable roles of robotic devices.

\title{
How to reference
}

In order to correctly reference this scholarly work, feel free to copy and paste the following:

Ioannis Sarakoglou, Sophia Kousidou, Nikolaos G. Tsagarakis and Darwin G. Caldwell (2007). ExoskeletonBased Exercisers for the Disabilities of the Upper Arm and Hand, Rehabilitation Robotics, Sashi S Kommu (Ed.), ISBN: 978-3-902613-04-2, InTech, Available from: http://www.intechopen.com/books/rehabilitation_robotics/exoskeletonbased_exercisers_for_the_disabilities_of_the_upper_arm_and_hand

\section{INTECH}

open science | open minds

\author{
InTech Europe \\ University Campus STeP Ri \\ Slavka Krautzeka 83/A \\ 51000 Rijeka, Croatia \\ Phone: +385 (51) 770447 \\ Fax: +385 (51) 686166 \\ www.intechopen.com
}

\author{
InTech China \\ Unit 405, Office Block, Hotel Equatorial Shanghai \\ No.65, Yan An Road (West), Shanghai, 200040, China \\ 中国上海市延安西路65号上海国际贵都大饭店办公楼405单元 \\ Phone: +86-21-62489820 \\ Fax: +86-21-62489821
}


(C) 2007 The Author(s). Licensee IntechOpen. This chapter is distributed under the terms of the Creative Commons Attribution-NonCommercial-ShareAlike-3.0 License, which permits use, distribution and reproduction for non-commercial purposes, provided the original is properly cited and derivative works building on this content are distributed under the same license. 